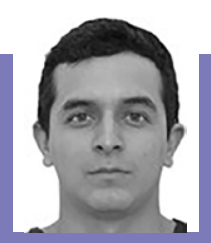

Dr Raul

Castaneda

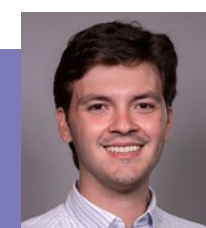

Dr Carlos

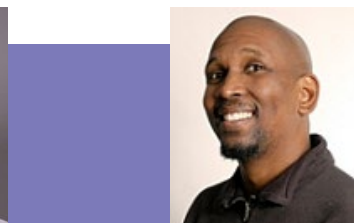

Dr Aaron

Buitrago-Duque L. Robinson

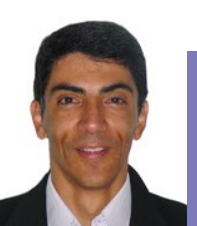

Dr Jorge GarciaSucerquia

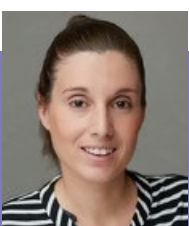

Dr Ana

Doblas

E: adoblas@memphis.edu T: +19016783650 W: https://oirl.github.io/Blind-PS-DHM-methods/

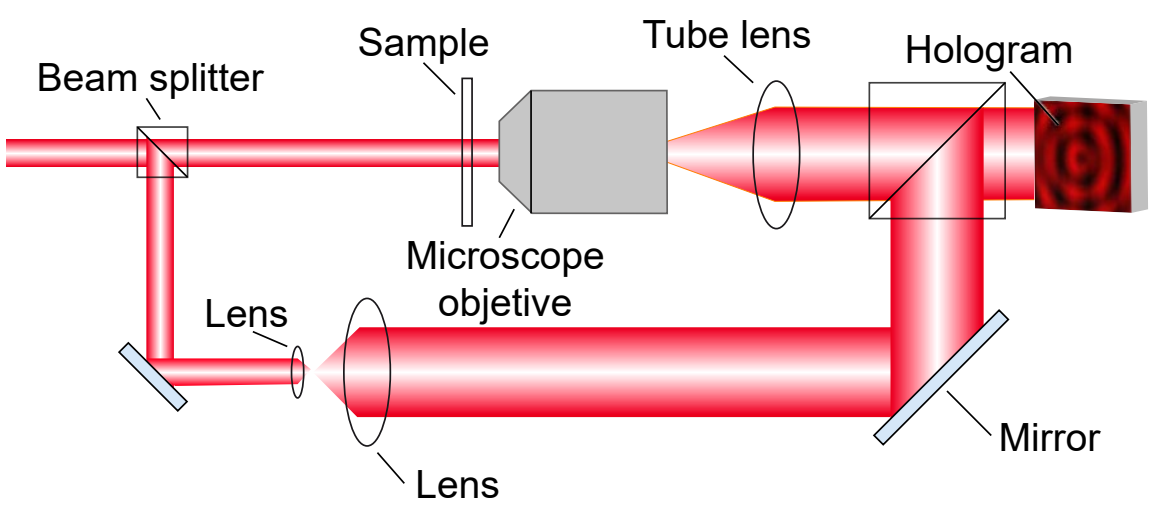

\title{
A New Computational Approach to Digital Holographic Microscopy
}

\section{Research Objectives}

The team is optimising PS-DHM technology to make it more suitable for dynamic imaging.

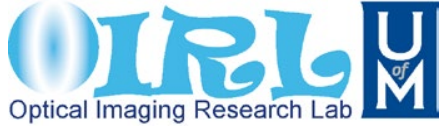

he also received his degree in Engineering Physics. He has been working with the Optics and Opto-Digital Processing group from this institution since 2016, developing his research interests in digital holographic microscopy, numerical modelling of physical systems, and optical metrology.

\section{Aaron L. Robinson is the Graduate} Coordinator for and an Associate Professor of the Department of Computer and Electrical Engineering at the University of Memphis. $\mathrm{He}$ is also member of the DRONES Research Cluster. His research includes sensor system analysis and design (with a focus on infrared wavelengths), turbulence mitigation, light propagation, and physically based degraded visual environment simulation.

Jorge Garcia-Sucerquia received his $\mathrm{PhD}$ in physics from the Universidad de Antioquia at Medellin-Colombia in 2003. From 2004 to 2006, he held a postdoctoral position at Dalhousie University, Halifax, Canada in working on digital lensless holographic microscopy. From 2012 to 2013, he was a visiting professor at the Universidad de Valencia, Spain. Currently, he is an associate professor at the School of Physics, Universidad Nacional de Colombia, Medellin-Colombia.

Ana Doblas received $B S, M S$, and $\mathrm{PhD}$ degrees in Physics from the University of Valencia, Spain. From 2010 to 2015, she was a Graduate Research Assistant with the 3D Imaging and Display Laboratory in Valencia. In 2015, she became Postdoctoral Researcher in the Optical Coherence Imaging Laboratory (University of North Carolina at Chapel Hill US). Since 2016, she is at the Department of Electrical and Computer Engineering at the University of Memphis, US. Since 2019, Doblas is Assistant Professor and principal investigator of the Optical Imaging Research Laboratory.

\section{Funding}

The University of Memphis

student from the School of Physics of the

Universidad Nacional de Colombia, where

\section{Personal Response}

- Doblas, A., Buitrago-Duque, C., Robinson, A., and GarciaSucerquia, J. (2019). Phase-shifting digital holographic microscopy with an iterative blind reconstruction algorithm. Applied Optics, 58(34), G311-G317.

- Castaneda, R., Buitrago, C., Garcia-Sucerquia, J., Robinson, A., and Doblas, A. (2020, June). Fast-iterative blind reconstruction algorithms for accurate quantitative phase images in phase-shifting digital holographic microscopy. In Digital Holography and ThreeDimensional Imaging, HTh5D-3, Optical Society of America.

- Castañeda, R., Buitrago-Duque, C., Garcia-Sucerquia, J., and Doblas, A. (2020). Fast-iterative blind phase-shifting digital holographic microscopy using two images. Applied Optics, 59(24), 7469-7476.
What advantages could be offered through your approach to PS-DHM when compared with more conventional microscopy techniques?

- Advantages include the reduced number of acquisition images, resulting in a more suitable approach for dynamic samples, as well as an automatic approach without prior knowledge of the user. The latter would allow researchers to investigate the samples without knowledge in DHM, leading to a commercial prototype. 
Informatics \& Technology | Ana Doblas

\section{A New Computational Approach to Digital Holographic Microscopy}

Digital Holographic Microscopy offers a unique way for researchers to precisely examine the 3D topography of microscopic objects. However, until now the capabilities of this technique have been limited by complications involving the complex computational method used. The by Dr Ana Doblas, and collaborators have constructed algorithms which can automatically reconstruct 3D topography without any user expertise. Their work could offer new opportunities for using the technique in research areas ranging from materials physics to biomedical imaging.

Researchers have a wealth of ter a microscope. By measuring how light amples, they can often determine the $3 \mathrm{D}$ topologies of microscopic objects precise levels of detail. However, these techniques are not always entirely suitable for imaging transparent objects, such as living cells or artificial crystals. Without any way for researchers to determine the precise positions of the features contained within these samples, their results are likely to be less reliable.

This problem has now created an incentive to develop techniques which can directly create $3 \mathrm{D}$ digital images of researchers can use 'Digital Holographic

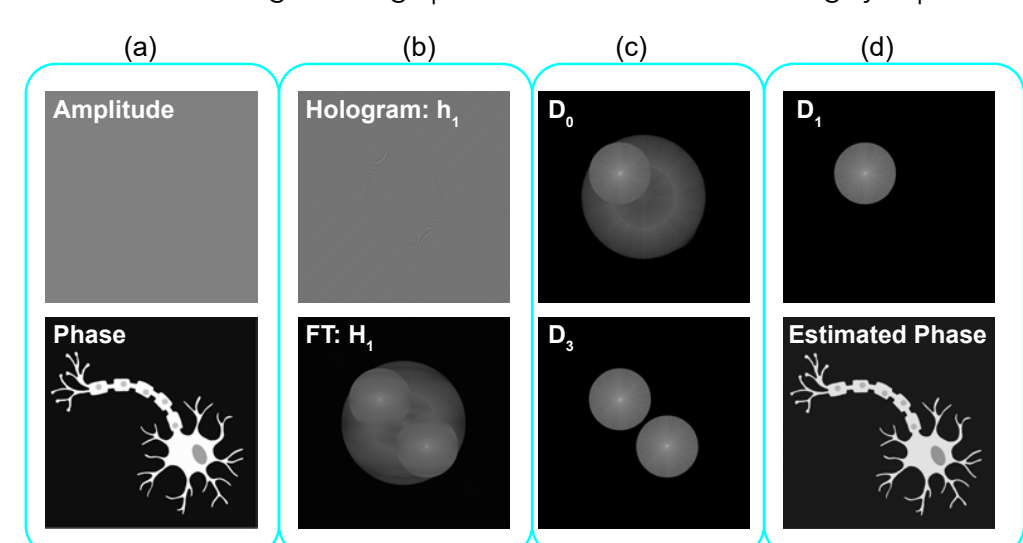

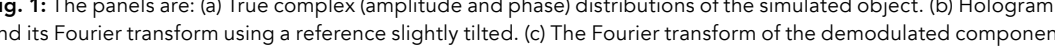

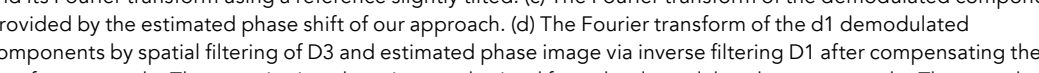

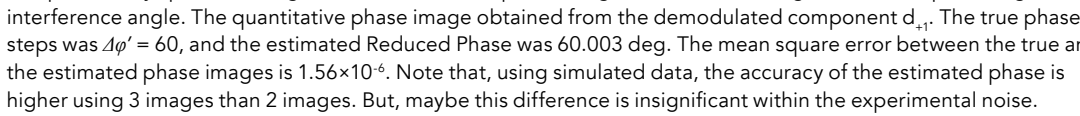
'object' and a 'reference' beam. As it interacts with the transparent sample, vaning degrees, depending on the dept to which it penetrated into the sample. Finally, these scattered wavefronts are collected by a microscope lens, and merged with the reference beam. can then calculate a transparent, $3 \mathrm{D}$ inage of the object using a specialised From these digital reconstructions researchers can precisely analyse the 3D structures contained within transparent the quapic objects. Currenty, however, reconstruns a high of the digital on the computational approach and the user's expertise in selecting the correct parameters, introducing an unavoidable source of human error. So far, this

(iDHM in cutting-edge studies, including An Anew compustato a DHM reconstruction

\section{OVERLAPPING ORDERS}

When a simple laser beam passes throug interference pattern is projected onto a screen, a bright peak in light intensity will appear in the centre of the pattern. where the beam would have shone if the grating were not there. However, other pairs of brightness peaks will also appear at regular intervals to either side of this central spot, whose widths, intensities, and spacings will vary both with the light's wavelength and the spacing between gaps in the grating. Subsequently, peak pairs at successively larger distances from the centre are described as having progressively higher 'orders' of diffraction.

Although the random speckled patterns recorded by DHM are far more complex by the same physical laws This abide that any peaks in brightness will be surrounded by three detectable orders of diffraction. Since microscopic target objects such as cells and crystal structures can have complex, arbitrary shapes, the paths taken by the light scattered off them will vary widely and unpredictably. Ultimately, this leads to substantial difficulties when interpreting their resulting interference patterns.

"The angle between the reference and object waves entails the possibility to operate under three different DHM architectures: in-line, slightly off-axis, or off-axis", explains DrAna Doblas, principal investigator of the Optical University of Memphis. "In slightly off- axis and in-line DHM systems, the three diffraction orders that compose the Without any way of knowing the extent to which this overlapping occurs, it is impossible for algorithms to reconstruct impossible for algorthms to reconstruct
images effectively. So far, accounting for this problem has required a cumbersom set of techniques.

STRUGGLES WITH PHASE-SHIFTING the same frequency are said to be 'phase shifted' if their points of highest and lowest amplitude are misaligned as they propagate. If two such waves are propagating together, they will destructively interfere with each other to reduce the light intensity seen by an observer - to an extent which depends on just how out of phase they are. On the wavelengths of visible light this principle can be exploited to determine precise distances.

Through the technique of Phase-Shifting DHM (PS-DHM), images with different phase shifts can be created alongside the main image. From these resulting 'phase maps', reconstruction algorithms can better calculate distances from the cam to different parts of the target object enabling more accurate $3 \mathrm{D}$ images. To an extent, PS-DHM allows researchers to mitigate the effects of overlapping diffraction orders, but the approach comes with its own limitations. "Phaseshifting techniques are needed to recover need for mut " DrDoblas describes "Conventional ، PS-DHM methods require the accurate knowledge of the phase shifts between the recorded holograms. However, experimentally such accuracy is frequenty arduous, leading to the use of inexact. values of the phase shift which produce distorted and unreliable phase maps."

In turn, image reconstruction algorithms are provided with a less reliable basis, leading to less accurate images and, subsequently, limiting the practicality of PS-DHM in the lab. Through their research, Dr Doblas and her collaborators aim to minimise the number of recording needed and to develop a more advanced set of algorithms to ease the use of
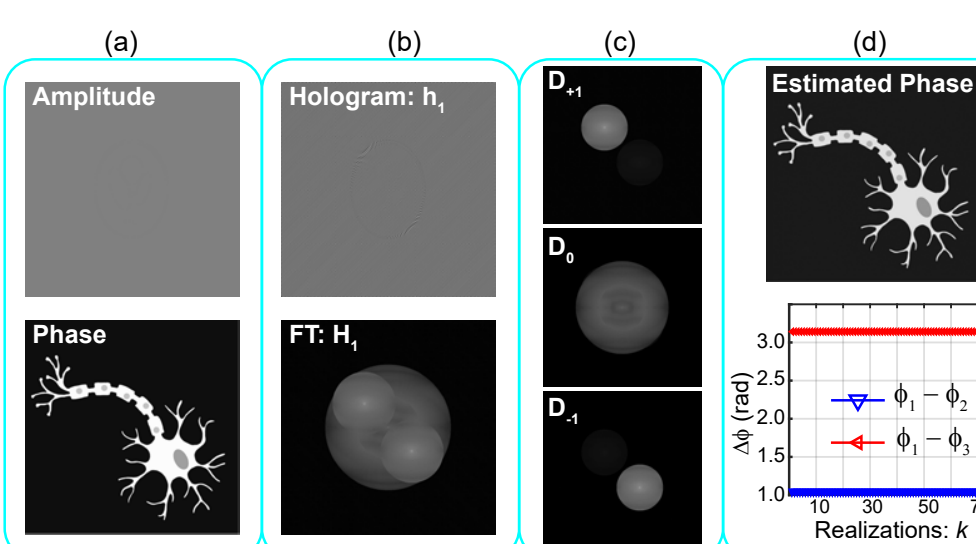
Fig. 2: The panels are: (a) True complex (amplitude and phase) distributions of the simulated object. (b) Hologram

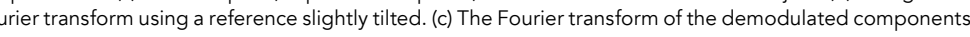

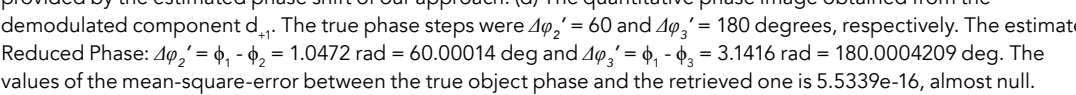

If Dr Doblas" techniques become more widely adopted, they could greatly improve the applicability of advanced microscopy.

\section{INCORPORATING}

BLIND ALGORITHMS

In a 2020 study, the research group take a more trial-and-error approach to phase shifting. These programs first take in mathematical approximations of hologram interference patterns, the they contrin recover the information longer be in unt heir outcomes can no longer be improved. This significantly holograms required for the process. "As an alternative of conventional PSDHM algorithms, blind phase-shifting algorithms do not need the accurate knowledge of phase shifts", Dr Doblas explains. "We present two phase-shifting algorithms for accurate quantitative phase images in PS-DHM. The proposed methods operate with at most three phase-shifted holograms."

Through their latest experiments, Dr Doblas' team has now clearly demonstrated four distinct advantages of their algorithms, compared with previous approaches to PS-DHM. Firstly, they can work effectively with a minimal number of recorded holograms since they don't require an accurate knowledge of phase shits. Secondly, hey produce a approaching just $0.005 \%$. Thirdly, the possible correlation between the phase shift of each image, and the phase of the actual image received fter processing. Finally, they require signicantly lower processing time

\section{BRINGING DHM TO THE LAB} Thanks to these numerous benefits, Dr Doblas and her collaborators nowhope of complex microscopic systems uhis move and change shape ortime. "The hallmarks of our methods are accuracy. and reduced acquistion and processing times leading to a PS-DHM system more suitable for dynamic imaging, as is the case of live cell imaging and colloidal systems", she concludes.

If Dr Doblas' techniques become more widely adopted, they could greatly improve the applicability of an advanced microscopy technique which has, until mecenty, been viewed as impractical by

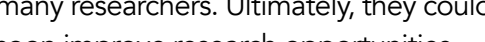
for groups ranging fromporisties studying the intricate physicists advanced artificite cystal structures of maging the time-varying behaviours of microscopic living systems. 


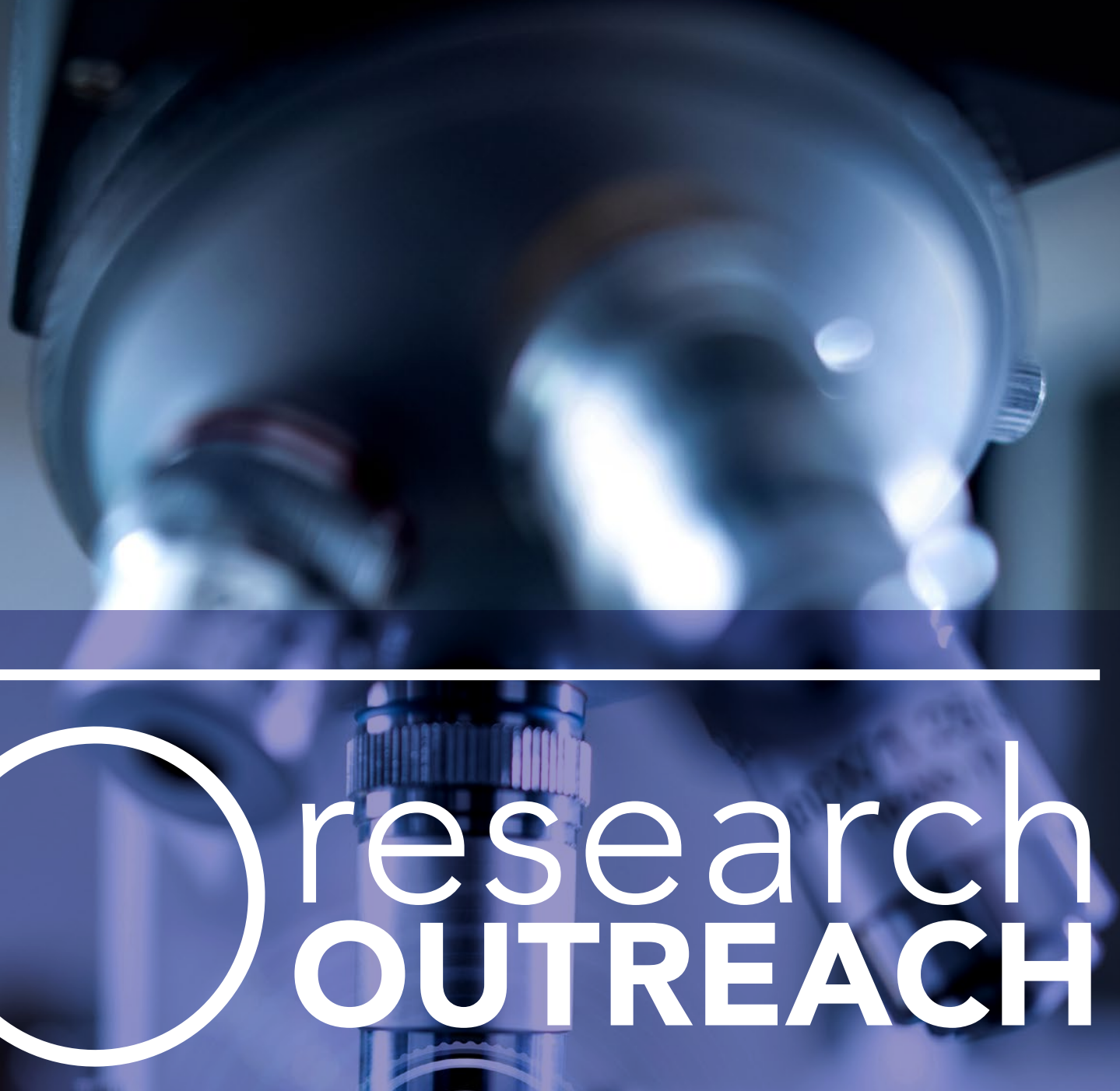

The public outreach magazine for the research community

\section{researchoutreach.org}

Partnership enquiries: simon@researchoutreach.org

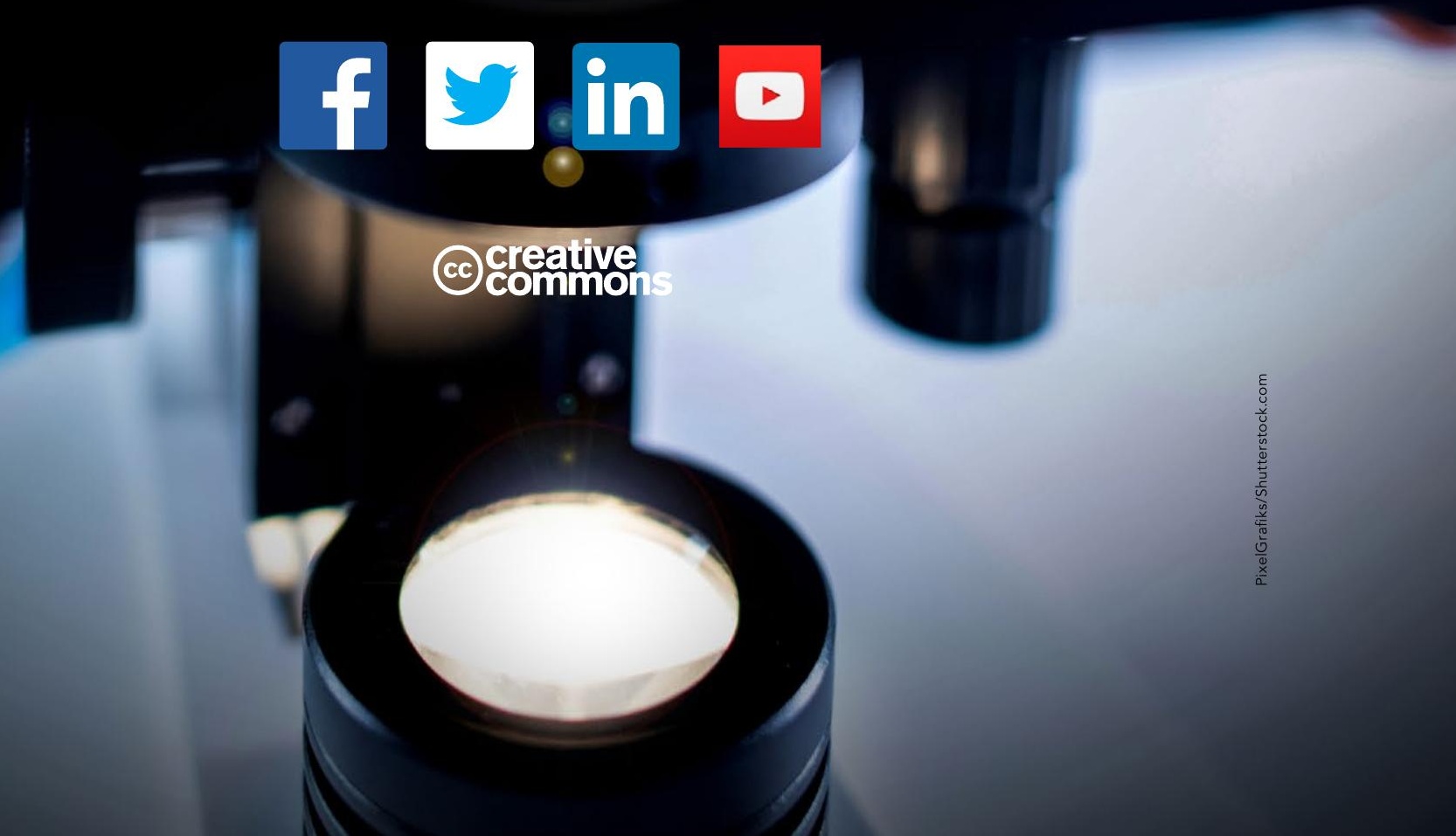

